\title{
TSC2 Gene Inactivation
}

National Cancer Institute

\section{Source}

National Cancer Institute. TSC2 Gene Inactivation. NCI Thesaurus. Code C36696.

A mutation in the TSC2 gene that either inhibits the expression of or results in the translation of an inactive tuberin protein. 